\title{
Chemical accelerator studies of isotope effects on collision dynamics of ion-molecule reactions: $\mathrm{Kr}^{+}+\mathrm{HD}$
}

\author{
S. Chivalak and P. M. Hierl \\ Department of Chemistry, University of Kansas, Lawrence, Kansas 66045 \\ (Received 14 May 1976; revised paper received 17 February 1977)
}

\begin{abstract}
The reaction of $\mathrm{Kr}^{+}$with $\mathrm{HD}$ has been studied as a function of relative collision energy over the range $0.08-3.1 \mathrm{eV}$ (c.m.) by measuring integral reaction cross sections and the velocity vector distributions of product ions formed when a collimated, energy selected beam of $\mathrm{Kr}^{+}$impinges on $\mathrm{HD}$ under single collision conditions. The ratio $\sigma\left(\mathrm{KrH}^{+}\right) / \sigma\left(\mathrm{KrD}^{+}\right)$passes through a sharp maximum $(\simeq 2.5)$ at about $0.7 \mathrm{eV}$ relative collision energy and decreases by a factor of 10 at higher energies. The isotopic product velocity vector distributions show a high but not perfect degree of symmetry about the center of mass at low energies but are extremely anisotropic at high energies, with the $\mathrm{KrH}^{+}$being strongly forward scattered and the $\mathrm{KrD}^{+}$being back scattered
\end{abstract}

\section{INTRODUCTION}

The effect of isotopic substitution upon the mechanisms and the rates of even the simplest chemical reactions remains a problem of great interest and considerable importance to the chemist. ${ }^{1}$ One aspect of this problem which has received increased attention in recent years has been the study of isotope effects upon reactions occurring at hyperthermal energies. ${ }^{2}$ A major portion of these studies have centered upon ion-molecule reactions, ${ }^{1,3,4}$ with the energy dependence of intramolecular isotope effects having been studied most thoroughly for the reaction:

$$
\mathrm{Ar}^{+}+\mathrm{HD}-\left[\begin{array}{l}
\mathrm{ArH}^{+}+\mathrm{D} \\
\mathrm{ArD}^{+}+\mathrm{H} .
\end{array}\right.
$$

Experimental methods employed have included highpressure mass spectrometry, ${ }^{5-7}$ ion cyclotron resonance, ${ }^{8}$ and chemical accelerator (i.e., tandem mass spectrometric or molecular beam) techniques. ${ }^{9-13}$ The results show that Reaction (1) proceeds by a direct mechanism over the entire energy range studied $(0.1-10$ eV) and that the kinetic isotope effect (KIE), expressed as the intensity ratio $\mathrm{ArH}^{+} / \mathrm{ArD}^{+}$, increases monotonically with collision energy. Theoretical explanations for these results have been attempted, using various mechanistic models, ${ }^{6,7,9,14-17}$ phase-space theory, ${ }^{16,18}$ and trajectory calculations. ${ }^{19,20}$

The corresponding reaction of the next heaviest rare gas ion,

$$
\mathrm{Kr}^{+}+\mathrm{HD}-\left[\begin{array}{l}
\mathrm{KrH}^{+}+\mathrm{D} \\
\mathrm{KrD}^{+}+\mathrm{H},
\end{array}\right.
$$

was first studied by Klein and Friedman, ${ }^{6}$ who used highpressure mass spectrometry to measure the $\mathrm{KrH}^{+} / \mathrm{KrD}^{+}$ ratio as a function of the repeller voltage (i.e., average reactant translational energy). They found that the ratio increased monotonically from a value of 0.81 at an average laboratory (LAB) collision energy of $0.4 \mathrm{eV}$, to a value of 1.12 at an average collision energy of $8.1 \mathrm{eV}$ (LAB). Henchman and co-workers, ${ }^{12}$ using an ion beam technique to measure the KIE as a function of reactant translational energy up to $20 \mathrm{eV}$ (LAB), made the remarkable discovery that the ratio $\mathrm{KrH}^{+} / \mathrm{KrD}^{+}$reached a maximum of about 2.5 at $16 \mathrm{eV}$ (LAB) and decreased at higher energies. As previously communicated, ${ }^{12}$ studies in our laboratory which extended to nearly $100 \mathrm{eV}$ essentially confirmed these earlier results and also demonstrated the existence of an inverse $\mathrm{KIE}\left(\mathrm{KrH}^{+} / \mathrm{KrD}^{+}<1\right)$ at collision energies greater than about $45 \mathrm{eV}$ ( $\mathrm{LAB}$ ) or $1.5 \mathrm{eV}$ in the center-of-mass (c. m.) system.

The present paper describes these measurements in detail and reports velocity vector distributions for the ionic products of Reaction (2) as a function of initial translational energy $T$ over the range $0.08-3.1 \mathrm{eV}$ (c. m.).

The reaction of $\mathrm{Kr}^{+}$with $\mathrm{H}_{2}$ (or $\mathrm{D}_{2}$ ) has, in itself, been the subject of considerable interest in the past several years. Although the reaction is exothermic by about 0.3 $\mathrm{eV}$ for the ground state reactants, a barrier to reaction of $0.44-1.2 \mathrm{eV}$ was predicted by Kuntz and Roach, ${ }^{22}$ who employed a semiempirical diatomics-in-molecules technique to calculate a potential energy surface for the ground state of collinear $\mathrm{KrH}_{2}^{+}$. Measurements of the differential cross section for scattering of $\mathrm{H}_{2}^{+}$on $\mathrm{Kr}$ led Henglein ${ }^{23}$ to estimate an activation energy of $0.7 \mathrm{eV}$ for the reaction of $\mathrm{Kr}^{+}$with $\mathrm{H}_{2}$. However, recent measurements ${ }^{24-26}$ of the rate coefficient for the reaction

$$
\mathrm{Kr}^{*}\left({ }^{2} P_{3 / 2}\right)+\mathrm{H}_{2} \rightarrow \mathrm{KrH}^{+}+\mathrm{H}
$$

near thermal energies have indicated that the activiation energy can be no larger than $0.05 \mathrm{eV}$. Beam studies ${ }^{27}$ of the reaction $\mathrm{Kr}^{+}\left(\mathrm{D}_{2}, \mathrm{D}\right) \mathrm{KrD}^{+}$reveal that the cross section is about 10 times smaller than that for the corresponding reaction of $\mathrm{Ar}^{+}$with $\mathrm{D}_{2}$. Product velocity vector distributions were found to be nearly isotropic about the center of mass at a relative translational energy of $0.89 \mathrm{eV}$ but to be forward peaked at nearly the spectator stripping velocity when the collision energy was increased to $2.70 \mathrm{eV}$ (c. m.). Studies in our laboratory of the reaction $\mathrm{Kr}^{+}\left(\mathrm{H}_{2}, \mathrm{H}\right) \mathrm{KrH}^{+}$yielded similar results. ${ }^{28}$

\section{EXPERIMENTAL}

\section{A. Apparatus}

The instrument used in this study is a single beamcollision chamber type of chemical accelerator with product velocity and angular analysis. $\mathrm{Kr}^{+}$ions, formed by impact of $35 \mathrm{eV}$ electrons, are focused into a nearly monoenergetic beam of variable energy $(0.5-100 \mathrm{eV}$ LAB) by a system of electrostatic lenses. This colli- 
mated beam passes through the collision chamber containing the $\mathrm{HD}$ at a pressure of about $10^{-3}$ tor $\mathrm{r}$ (i. e., under single collision conditions). The ion gun can be rotated about the center of the collision chamber, permitting the fixed detector to measure scattered products at various angles. Those ions leaving the collision chamber at the selected angle pass through a rectangular detection slit, a parallel plate retarding potential energy analyzer, and a set of strong focusing quadrupole lenses. Mass analysis of the ions is performed with the $30 \mathrm{~cm}$, 90 deg deflection magnetic sector analyzer of a Nuclide mass spectrometer from which the conventional ion source and accelerating electrodes have been removed. The individual components of this instrument have been described in detail elsewhere. ${ }^{29}$

\section{B. Internal states of the reactants}

Because the $\mathrm{Kr}^{+}$is produced by impact of $35 \mathrm{eV}$ electrons, the primary ion beam contains no doubly charged ions and about $1.5 \%$ of high energy metastable ions. ${ }^{30}$ Nearly all $(98.5 \%)$ of the $\mathrm{Kr}^{+}$ions, therefore, are in the ${ }^{2} P$ state and are presumably distributed statistically in a $2: 1$ ratio between the $J=\frac{3}{2}$ and the $J=\frac{1}{2}$ levels, $^{31}$ which differ in energy by $0.66 \mathrm{eV} .^{32}$

The neutral target gas is assumed to be in thermal equilibrium, so that the internal energy of the HD is determined by its temperature $\left(85^{\circ} \mathrm{C}\right.$ under the conditions: of the experiment). Nearly all of the HD molecules, therefore, are in their ground vibrational state. The most probable rotational state is $J=1$, which corresponds to an energy of about $0.011 \mathrm{eV}$. Consequently, most of the energy available to the reaction products is derived from the heat of reaction plus the initial translational energy in the center-of-mass (c. m. ) system.

\section{Heat of reaction}

Intermolecular potentials for $\mathrm{KrH}^{+}$have been derived from experiments on the elastic scattering of protons on krypton. Weise etal. ${ }^{332}$ obtained a value of $4.45 \pm 0.10$ $\mathrm{eV}$ for the well depth $D_{e}$, while Rich etal. ${ }^{33 \mathrm{~b}}$ reported a value of $4.6 \mathrm{eV}$. Assuming that the zero point vibrational energy of $\mathrm{KrH}^{+}$is $0.16 \mathrm{eV}$, equal to that of the isoelectronic molecule $\mathrm{HBr},{ }^{34}$ one obtains for the dissociation energy $D_{0}\left(\mathrm{Kr}-\mathrm{H}^{+}\right)$values of 4.29 and $4.44 \mathrm{eV}$ from the two scattering experiments. These values are in good agreement with the value of $4.38 \mathrm{eV}$ recently obtained from flowing afterglow studies of the equilibrium between $\mathrm{KrH}^{+}$and $\mathrm{H}_{3}^{+}$in $\mathrm{Kr}-\mathrm{H}_{2}$ mixtures. ${ }^{35}$ Using the latter value for $D_{0}\left(\mathrm{Kr}-\mathrm{H}^{+}\right)$and taking $D_{0}(\mathrm{H}-\mathrm{H})=4.51 \mathrm{eV},{ }^{36}$ Reaction (3) is exothermic by $0.3 \pm 0.1 \mathrm{eV}$ when the $\mathrm{Kr}^{+}$ is in the ${ }^{2} P_{3 / 2}$ ground state.

\section{Doppler effects}

The data analysis presented here was performed under the customary assumption of monoenergetic ions colliding with stationary target molecules (i.e., the spread in projectile velocities and the thermal motion of the target gas molecules have been ignored). In the lowest collision energy experiment $(0.08 \mathrm{eV}$ c. m. ), however, the velocity of the reactant ion is only about twice the most probable speed of the neutral reactant.
The most serious consequence of the thermal motion of the neutral reactant is to cause collisions to occur with a distribution of relative velocities in a given experiment. As shown in Fig. 1, the resulting spread in relative collision energies is much greater than that caused simply by the spread in projectile velocities: Thus, the energy resolution achieved in the present experiments is limited by the Doppler broadening caused by the thermal motion of the target molecules rather than by the energy spread of the reactant beam.

The principal effect of this spread in collision energies will manifest itself in the measurements of the reaction cross sections and, more particularly, of the kinetic isotope effect. The effective cross section $\sigma_{\text {orf }}$ measured experimentally is given by the integral ${ }^{37}$

$$
\sigma_{\text {eff }}=\int_{0}^{\infty}\left(T / T_{0}\right)^{1 / 2} \sigma(T) f\left(T_{0}, T\right) d T,
$$

where the factor $\left(T / T_{0}\right)^{1 / 2}$ is proportional to the effective path length, $\sigma(T)$ is the true cross section at collision energy $T$, and $f\left(T_{0}, T\right)$ is the distribution function in relative collision energies [see Eq. (11) of Ref. 37]. $T_{0}$ is the nominal and $T$ the actual c. $m$. collision energy. This averaging process will tend to smear out any structure in the excitation function (cross section as a function of collision energy) for the reaction under study. Consequently, the unusual low energy kinetic isotope effect reported here is actually considerably larger and much more sharply peaked than is indicated by the present data, which have not been corrected for Doppler broadening.

On the other hand, the kinematic data (product angular and velocity distributions) are much less strongly af-

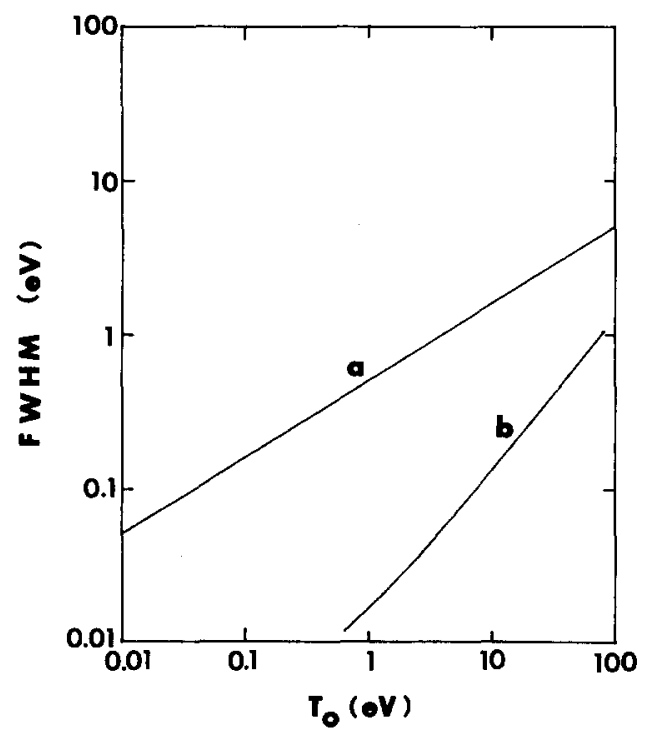

FIG. 1. Full-width at half-maximum, FWHM, of the distribution of relative (i.e., center-of-mass) collision energies vs $T_{0}$, the nominal c.m. collision energy. Curve $a$ represents the width, calculated from Eq. (A1), resulting from the interaction of a monoenergetic $\mathrm{Kr}^{+}$beam with $\mathrm{HD}$ which has an isotropic velocity distribution corresponding to a temperature of $358^{\circ} \mathrm{K}$. Curve $b$, calculated from Eq. (A3), represents the width of the energy distribution that would be caused (in the absence of target motion) by the spread in energy of the $\mathrm{Kr}^{+}$beam. 


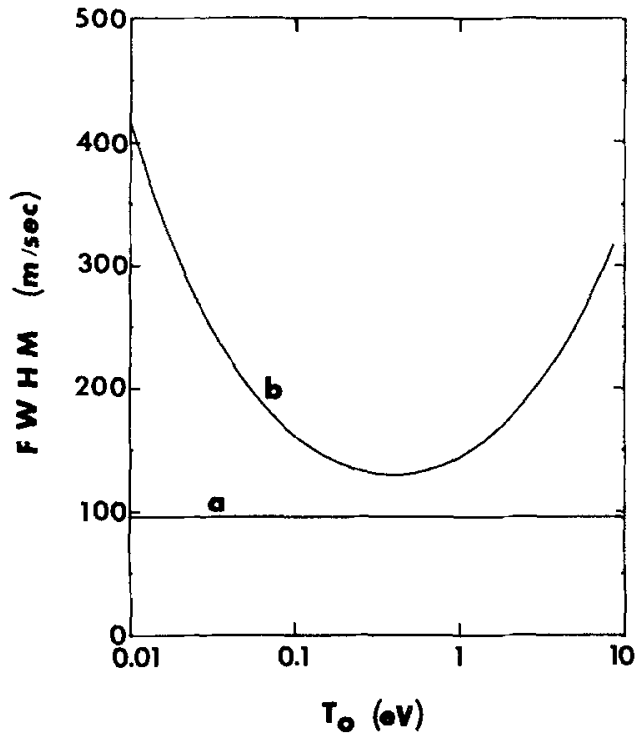

FIG, 2. Full-width at half-maximum, FWHM, of the distribution of centroids for the $\mathrm{Kr}^{+}+\mathrm{HD}$ sy stem, vs $T_{0}$, the nominal c. m. collision energy. Curve $a$, calculated from Eq. (A5), indicates the broadening due solely to thermal motion of the HD target molecules. Curve $b$, calculated from Eq. (A8), represents the broadening resulting from the spread in $\mathrm{Kr}^{+}$velocities.

fected by the target's thermal motion. Although nonzero target velocities will change the velocity of the c.m. of the system from its nominal value, the great disparity between target and projectile masses minimizes the target's contribution to the total momentum of the system. Consequently, the target's thermal motion makes a smaller contribution to the width of the centroid distribution than does the spread in projectile velocities (see Fig. 2). Since the projectile's velocity spread is itself small compared to the width of the product velocity distribution (see Figs 3-10), the product velocity vector distributions are relatively unaffected by thermal motion of the target molecules.

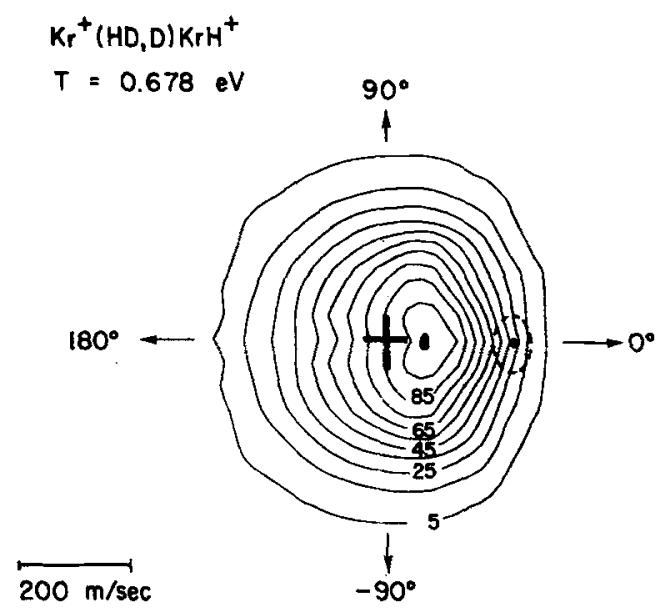

FIG. 3. Velocity vector distribution for $\mathrm{KrH}^{+}$formed in Reaction (2a) at the collision energy $T=0,678 \mathrm{eV}$ (c.m.). The $\mathrm{KrH}^{+}$ intensities, normalized to 100 at the position of maximum intensity, are shown relative to the Cartesian system, $P_{C}(u, \theta)$. Arrows represent scattering angles $(\theta)$ with respect to the center of mass, which is marked by the heavy cross. The dashed oval represents the $20 \%$ contour line for the reactant $\mathrm{Kr}^{+}$ion beam.

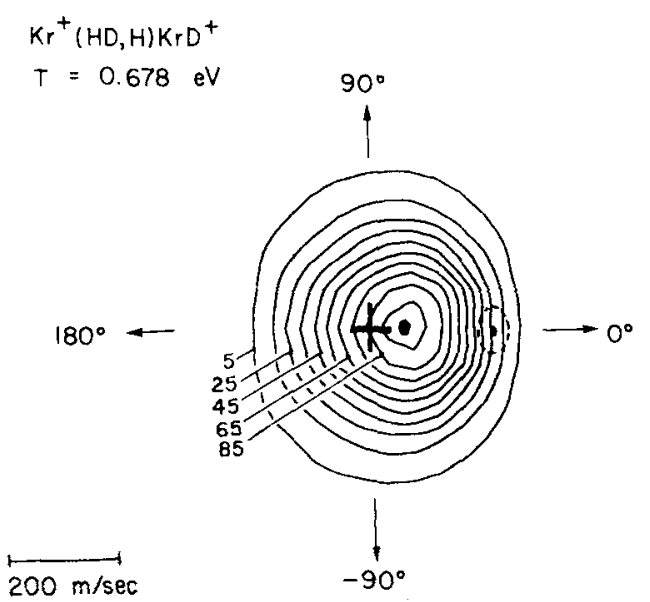

FIG. 4. Velocity vector distribution $P_{C}(u, \theta)$ for $\mathrm{KrD}^{+}$formed in Reaction (2b) at the collision energy $0.678 \mathrm{eV}$ (c.m.). Note the similarity of this product distribution to that measured for $\mathrm{KrH}^{+}$produced at the same collision energy ( $\mathrm{F}$ ig. 3 ).

Because this motion makes only a small contribution to the product angular distributions, and because the reaction cross sections are obtained in any case by integra. tion over the entire product angular distribution, target motion should have no effect upon product collection efficiency. The observed low energy kinetic isotope effect is, therefore, real and not an experimental artifact arising from target motion. On the contrary, the actual effect of the target motion has been to make this isotope effect appear smaller and less sharply peaked than it truly is.

\section{RESULTS}

\section{A. Kinetic isotope effect}

Integral reaction cross sections $\sigma_{R}$ are calculated from the familiar "thin target" approximation

$$
\begin{aligned}
& \mathrm{Kr}^{+}(H D, D) \mathrm{KrH}^{+} \\
& T=1.41 \mathrm{eV}
\end{aligned}
$$

$90^{\circ}$

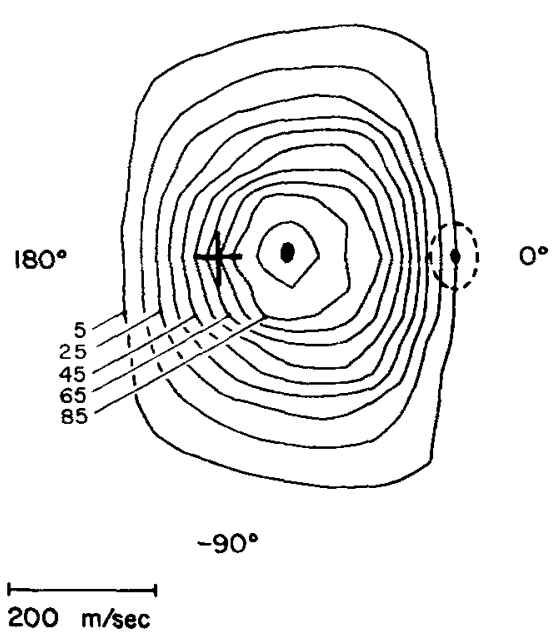

FIG. 5. Velocity vector distribution $P_{C}(u, \theta)$ for $\mathrm{KrH}^{+}$formed in Reaction (2a) at the collision energy $T=1.41 \mathrm{eV}$ (c. m.). Note that the position of maximum $\mathrm{KrH}^{+}$intensity has shifted forward of the center of mass (heavy cross). 


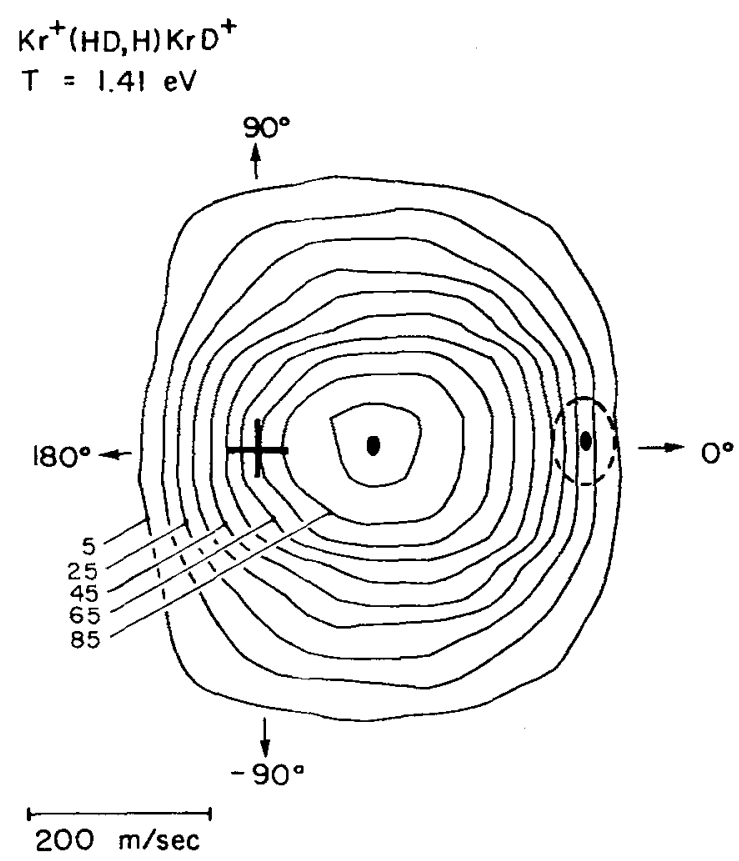

FIG. 6. Velocity vector distribution $P_{C}(u, \theta)$ for $\mathrm{KrD}^{+}$formed in Reaction (2b) at the collision energy $T=1.41 \mathrm{eV}(\mathrm{c}, \mathrm{m}$.$) . The$ position of maximum $\mathrm{KrD}^{*}$ intensity has shifted forward of the center of mass (heavy cross), as was found for the $\mathrm{KrH}^{+}$product ion at this collision energy (Fig. 5 ).

$$
\sigma_{R}=\frac{I_{\mathcal{C}} / I_{A}}{n_{B} L},
$$

where $I_{C}$ is the total reactively scattered product ion intensity, $I_{A}$ is the transmitted primary $\left(\mathrm{Kr}^{+}\right)$ion intensity, $n_{B}$ is the number density of HD molecules in the collision chamber, and $L$ is the collision path length. As before, ${ }^{29}$ the ratio of total ion intensities is given by

$$
\frac{I_{C}}{I_{A}}=\frac{I_{C}\left(0^{\circ}\right) K_{C}}{I_{A}\left(0^{\circ}\right) K_{A}} K_{1} K_{2}
$$

where $I_{C}\left(0^{\circ}\right)$ and $I_{A}\left(0^{\circ}\right)$ are the observed ion intensities at the angular maximum, $K_{C}$ and $K_{A}$ correct for differ-

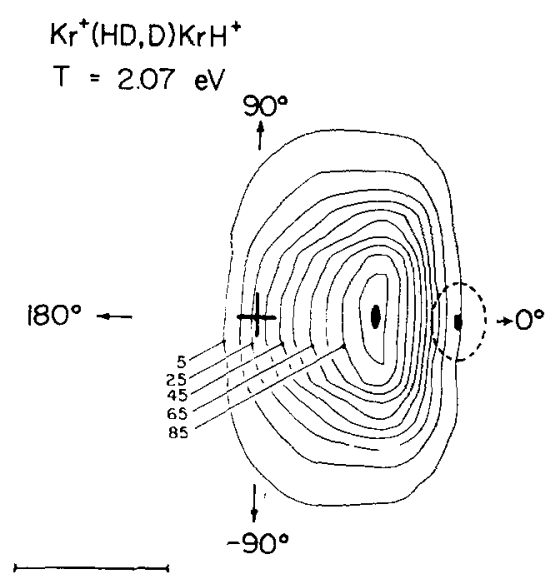

$300 \mathrm{~m} / \mathrm{sec}$

FIG. 7. Velocity vector distribution $P_{C}(u, \theta)$ for $\mathrm{KrH}^{+}$formed in Reaction (2a) at the collision energy $T=2.07 \mathrm{eV}$ (c.m.). At this energy, the $\mathrm{KrH}^{+}$is strongly forward peaked, with the position of maximum intensity occurring at nearly the velocity predicted by the spectator stripping model.

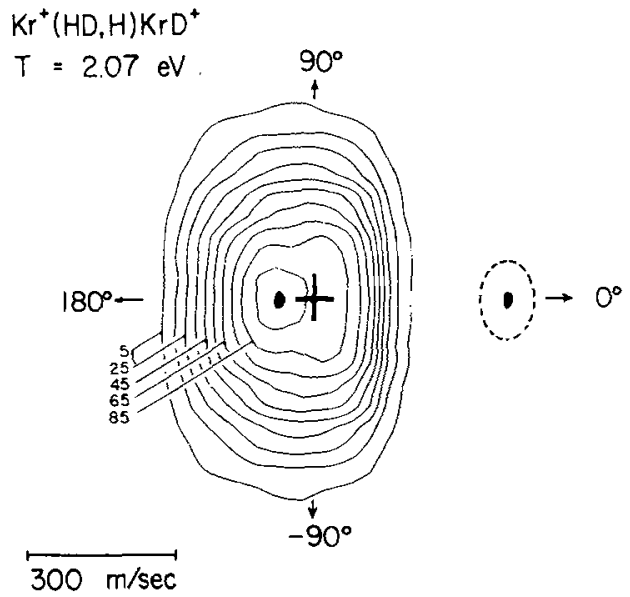

FIG. 8. Velocity vector distribution $P_{C}(u, \theta)$ for $\mathrm{KrD}^{+}$formed in Reaction (2b) at the collision energy $T=2.07 \mathrm{eV}$ (c. m.). Whereas the $\mathrm{KrH}^{+}$distribution was strongly forward peaked at this collision energy ( $\mathrm{Fig}$. 7), the $\mathrm{KrD}^{+}$product undergoes predominantly large-scale scattering, with the position of maximum intensity occurring behind the center of mass (heavy cross).

ences in collection efficiency caused by differences in angular distribution, $K_{1}$ corrects for differences in multiplier gain, and $K_{2}$ corrects for any broadening of the mass spectral peak of ions formed with a wide distribution of kinetic energies.

Because the relatively small cross sections for these reactions resulted in low product ion intensity, the quantities $K_{1}$ and $K_{2}$ could not be measured directly and were consequently assumed to equal unity. This assumption is consistent with results obtained in previous studies of other reactions and is not expected to introduce an error of more than a few percent. The correction factor $K_{C} /$

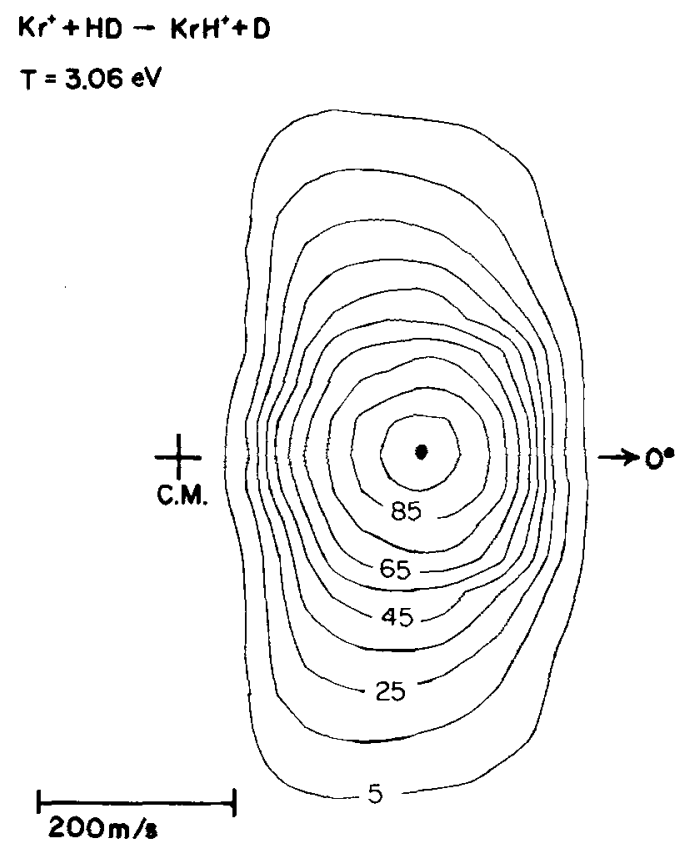

FIG. 9. Velocity vector distribution $P_{C}(u, \theta)$ for $\mathrm{KrH}^{+}$formed in Reaction (2a) at the collision energy $T=3.06 \mathrm{eV}$ (c. m.). The $\mathrm{KrH}^{+}$product is strongly forward peaked, with the position of maximum intensity occurring almost at the spectator stripping velocity. 


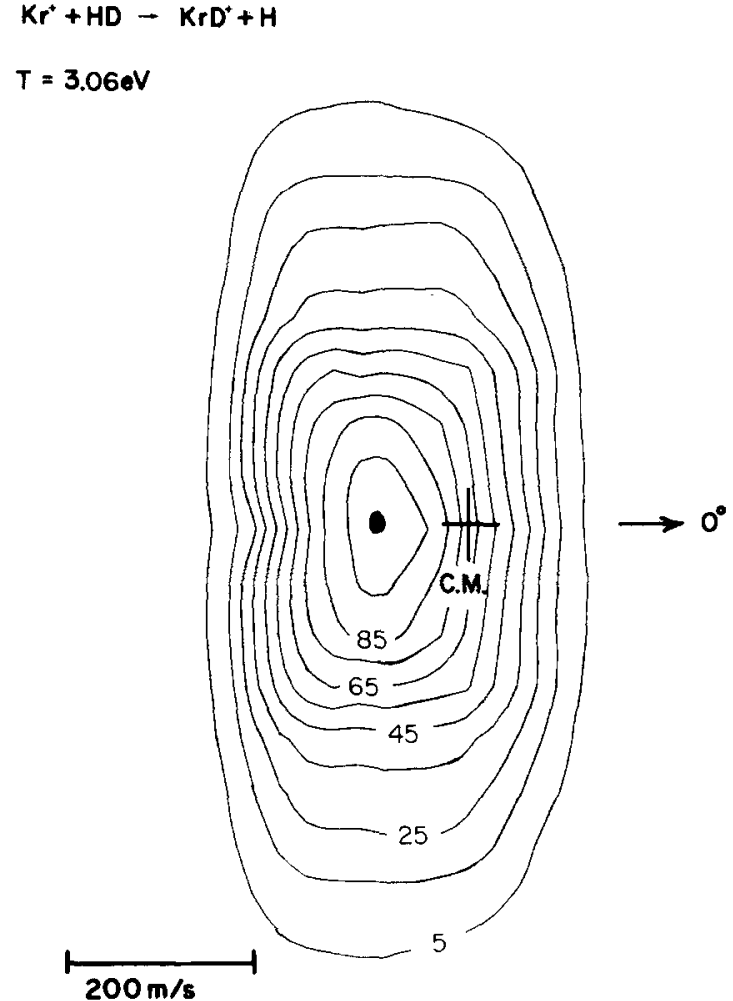

FIG. 10. Velocity vector distribution $P_{C}(u, \theta)$ for $\mathrm{KrD}^{+}$formed in Reaction (2b) at the collision energy $T=3.06 \mathrm{eV}$ (c.m.). The $\mathrm{KrD}^{+}$intensity is predominantly located in the backward hemisphere.

$K_{A}$ was obtained directly by integration of the measured laboratory angular distributions at each collision ener gy. ${ }^{28}$ The values obtained and the integral reaction cross sections calculated from Eq. (5) for Reactions (2a) and (2b) are listed in Table I. The experimental uncertainty in $\sigma_{R}$ is estimated to be $\pm 50 \%$.

The kinetic isotope effect (KIE), expressed as the ratio of cross sections $\sigma\left(\mathrm{KrH}^{+}\right) / \sigma\left(\mathrm{KrD}^{+}\right)$, is displayed as a function of collision energy in Fig. 11.

\section{B. Kinematic data}

Angular $(\Theta)$ distributions in the LAB system are obtained for the ionic species $\mathrm{Kr}^{+}, \mathrm{KrH}^{+}$, and $\mathrm{KrD}^{+}$by recording the appropriate ion signal while rotating the ion gun about the center of the collision chamber. Because the detector views a decreasing fraction of the collision path length with increasing scattering angle, the observed ion signal at each $L A B$ angle is divided by the path length subtended by the detector at that angle. ${ }^{29}$ The resulting angular distributions $I_{L}\left(\Theta, \Phi=0^{\circ}\right)$, which are normalized to unity at the angular maximum, represent the relative ion intensity scattered through a $\mathrm{LAB}$ angle $\Theta$ in the plane $\Phi=0^{\circ}$ from a reaction path of unit length.

The stopping potential curves obtained at various angles are first scaled to reflect the total relative intensity at that angle and are then differentiated to yield the energy distribution at that angle, $I_{L}\left(E, \Theta, \Phi=0^{\circ}\right)$. These energy distributions are next converted to velocity distributions by multiplying each point by the corresponding
TABLE I. Integral reaction cross sections for the system $\mathrm{Kr}^{+}$ $+\mathrm{HD} .^{\mathbf{a}}$

\begin{tabular}{|c|c|c|c|c|}
\hline $\begin{array}{l}\text { Most probable } \\
\text { relative } \\
\text { energy } \\
T(\mathrm{eV})\end{array}$ & $\begin{array}{l}\text { Pressure } \\
\text { of } \mathrm{HD} \\
\left(10^{-3} \text { torr }\right)\end{array}$ & $\begin{array}{l}\text { Cross section } \\
\text { for } \mathrm{KrH}^{+} \\
\text {formation } \\
\sigma\left(\mathrm{KrH}^{+}\right)\left(\AA^{2}\right)\end{array}$ & $\begin{array}{l}\text { Cross section } \\
\text { for } \mathrm{KrD}^{+} \\
\text {formation } \\
\sigma\left(\mathrm{KrD}^{+}\right)\left(\AA^{2}\right)\end{array}$ & $\begin{array}{l}\text { Kinetic } \\
\text { isotope } \\
\text { effect } \\
\sigma\left(\mathrm{KrH}^{+}\right) \\
\sigma\left(\mathrm{Kr}^{+} \mathrm{D}^{+}\right) \\
\end{array}$ \\
\hline 0.08 & $1: 10$ & 2.55 & 1.99 & 1.28 \\
\hline 0.24 & 1.05 & 3.16 & 1.97 & 1.60 \\
\hline 0.33 & 1.10 & 2.05 & 1.16 & 1.77 \\
\hline 0.51 & 1.18 & 1.62 & 0.81 & 2.00 \\
\hline 0.52 & 1.20 & 1.95 & 1.00 & 1.95 \\
\hline 0.67 & 1.08 & 1.55 & 0.63 & 2.45 \\
\hline 0.70 & 1.15 & 1.68 & 0.75 & 2.24 \\
\hline 0.86 & 1.18 & 2.03 & 0.92 & 2.21 \\
\hline 1.04 & 1.09 & 1.77 & 0.93 & 1.90 \\
\hline 1.08 & 1.09 & 1.62 & 0.90 & 1.80 \\
\hline 1.26 & 1.39 & 0.95 & 0.60 & 1.60 \\
\hline 1.35 & 1.20 & 0.87 & 0,70 & 1.25 \\
\hline 1.47 & 1.22 & 1.22 & 1.03 & 1.18 \\
\hline 1.75 & 1.10 & 0.58 & 0.67 & 0.86 \\
\hline 2.02 & 1.30 & 0.58 & 1.01 & 0.58 \\
\hline 2.39 & 1.32 & 0.26 & 0.41 & 0.64 \\
\hline 2.72 & 1.22 & 0,12 & 0.44 & 0.27 \\
\hline 3.06 & 1.59 & 0.16 & 0.61 & 0.27 \\
\hline
\end{tabular}

aThe $\mathrm{Kr}^{+}$ions, produced by impact of $35 \mathrm{eV}$ electrons, are presumably distributed statistically in a 2:1 ratio between the ${ }^{2} P_{3 / 2}$ and ${ }^{2} P_{1 / 2}$ states.

bThe most probable relative energy, $T$, is calculated from the peak in the $\mathrm{Kr}^{+}$laboratory energy distribution, $E_{\mathrm{LAB}}\left(\mathrm{Kr}^{+}\right)$. The target molecules are assumed to be stationary.

LAB velocity $v$ in accord with the transformation ${ }^{38}$

$$
I_{L}(E, \Theta, \Phi) d E d \Omega=I_{L}(v, \Theta, \Phi) d v d \Omega \text {. }
$$

These LAB cross sections, $I_{L}(v, \Theta, \Phi)$ are converted to probabilities in Cartesian velocity space according to the transformation

$$
P_{C}\left(v_{x}, v_{y}, v_{k}\right)=P_{C}\left(u_{x}, u_{y}, u_{z}\right)=v^{-2} I_{L}(v, \Theta, \Phi),
$$

where $P_{C}$ represents the probability of finding product in a given volume of velocity space and $u$ represents velocity in the $\mathrm{c} . \mathrm{m}$. coordinate system. ${ }^{39}$ There probabilities are then scaled, with the highest intensity arbitrarily set equal to 100 . These relative probabilities, displayed as

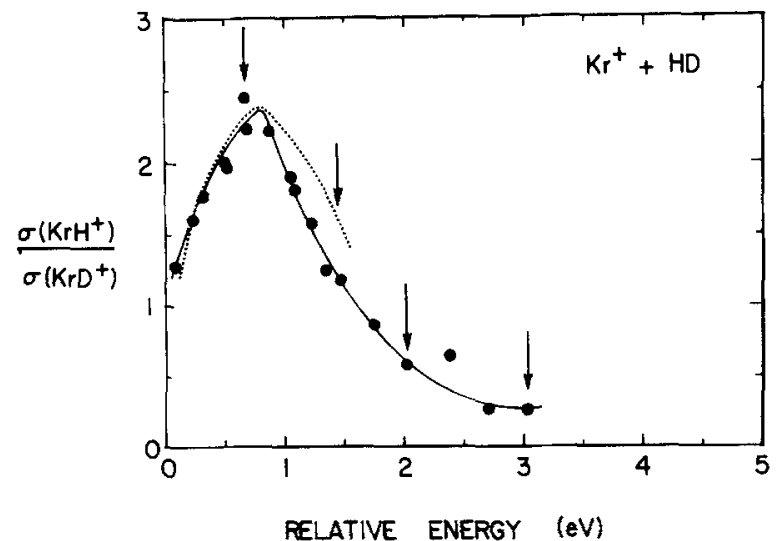

FIG. 11. Kinetic isotope effect, $\sigma\left(\mathrm{KrH}^{+}\right) / \sigma\left(\mathrm{KrD}^{+}\right)$, as a function of the relative collision energy (c. $m$. system) for the reaction of $\mathrm{Kr}^{+}$with $\mathrm{HD}$. The solid curve is a best fit to the present data (solid circles). The dotted line represents the results of Henchman and co-workers. ${ }^{12}$ The vertical arrows indicate the collision energies at which complete velocity vector distributions were obtained for both ionic products. 
contours of equal product intensity, are plotted on a velocity vector diagram for which the velocity of the center of mass of the system serves as the origin of a polar coordinate system. The distance of any point from the origin, therefore, represents the c. m. speed $(u)$ of the product ion, and the direction represents the c.m. scattering angle $(\theta)$ with respect to the relative velocity vector (in this case, simply the original direction of the reactant ion beam). The result is a map of relative intensities, $P_{c}(u, \theta)$, as seen by a detector located at c. $\mathrm{m}$. velocity $u$ and angle $\theta$, sensitive to particles in an element $d u_{x} d u_{y} d u_{z}$ of velocity space. Such velocity vector diagrams have been constructed for the formation of $\mathrm{KrH}^{+}$and $\mathrm{KrD}^{+}$at four different energies of the $\mathrm{Kr}^{+}$ion beam (Figs 3-10).

The angular information contained in these contour maps can be extracted by integration of $P_{C}$ over $u$ to obtain the relative polar differential reaction cross section $I(\theta) \sin \theta$, where

$$
I(\theta)=\int_{0}^{\infty} u^{2} P_{C}(u, \theta) d u .
$$

Center-of-mass angular distributions, obtained by plotting these differential cross sections vs the c. $m$. scattering angle $\theta$, are shown for $\mathrm{KrH}^{+}$and $\mathrm{KrD}^{+}$at four different energies of the $\mathrm{Kr}^{+}$ion beam in Figs. 12-15.

\section{DISCUSSION}

\section{A. Kinetic isotope effect}

As shown in Fig. 11, the ratio $\sigma\left(\mathrm{KrH}^{+}\right) / \sigma\left(\mathrm{KrD}^{+}\right)$has a value near unity at the lowest collision energy, passes through a maximum of nearly 2.5 at $T \cong 0.8 \mathrm{eV}$ (c. m.), and then decreases to a value of about 0.25 at $T \sim 3 \mathrm{eV}$. This finding is in excellent agreement with Henchman's results $^{12}$ over the energy range $0.1-1.5 \mathrm{eV}$, and is consistent with the low energy $(T<0,3 \mathrm{eV})$ data of Klein and Friedman. ${ }^{6}$

Both the sharp maximum at low energies $(T<1.5 \mathrm{eV})$ and the inverse isotope effect at high energies $(T>1.5$ $\mathrm{eV}$ ) are quite extraordinary and in marked contrast with the results reported by other workers for the analogous

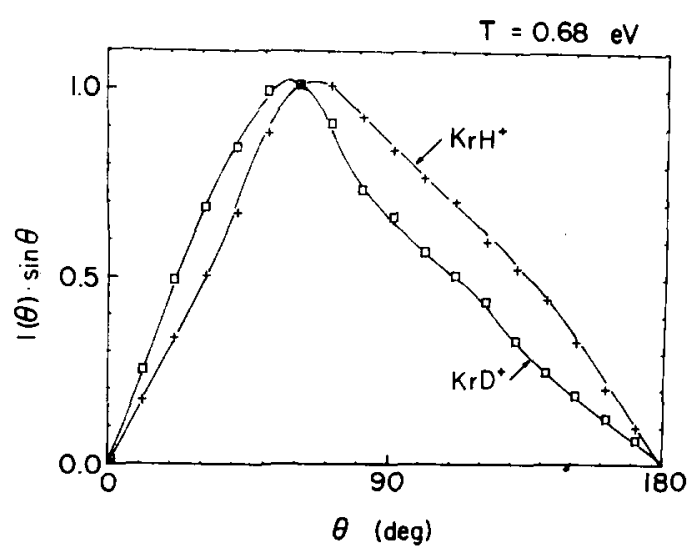

FIG. 12. Center-of-mass angular $(\theta)$ distributions for $\mathrm{KrH}^{+}$ (crosses) and $\mathrm{KrD}^{+}$(squares) produced in Reaction (2) at 0.678 $\mathrm{eV}$ initial relative energy. Note the similarly of the distributions for the two ieotopic products and the approximate (but not 'perfect) symmetry about $90^{\circ}$.

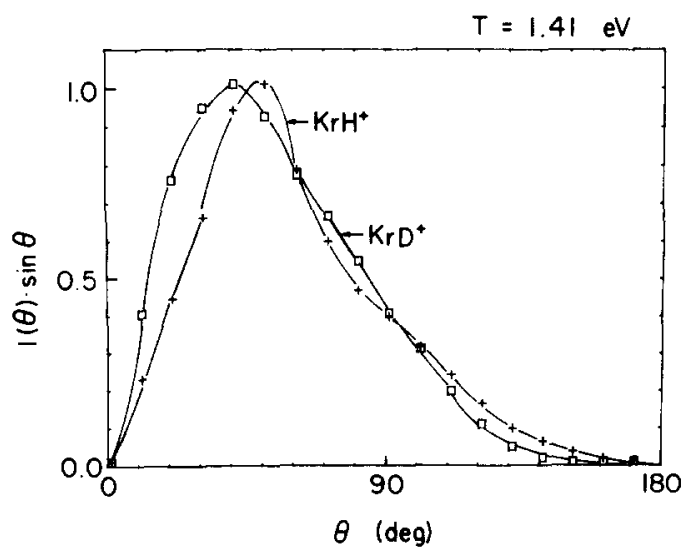

FIG. 13. Center-of-mass angular $(\theta)$ distributions for $\mathrm{KrH}^{+}$ (crosses) and $\mathrm{KrD}^{+}$(squares) produced in Reaction (2) at 1.41 $\mathrm{eV}$ initial relative energy. Again, note the similarity of the distributions.

reactions of $\mathrm{Ar}^{+}, \mathrm{N}_{2}^{+}, \mathrm{CO}^{+}$, and other simple ions with HD. ${ }^{10,12,40}$ In these cases, the KIE was near unity at the lowest collision energies and increased (slowly at first but then more rapidly) with increasing collision energy, reaching values greater than 2 only at rather high $>4$ $\mathrm{eV}$ ) collision energies. These results have been discussed in terms of complex formation, ${ }^{6}$ energy and momentum conservation, ${ }^{40,41}$ potential energy surfaces, ${ }^{10,20,43}$ and various kinematic models. ${ }^{9,11,14-17,42}$

The reaction of $\mathrm{O}^{+}$with $\mathrm{HD}$, however, reportedly ${ }^{44}$ exhibits an energy-dependent KIE quite similar to that shown here for the reaction of $\mathrm{Kr}^{+}$with $\mathrm{HD}$.

We have formulated a simple "orientation probability" model (see Paper II of this series, following article) which reproduces quite the observed KIE over the energy range $0.1-1.5 \mathrm{eV}(\mathrm{c} . \mathrm{m}$.). This model is based upon the fact that in the HD molecule the center of mass is displaced from the center of polarizability, thus causing the intermolecular potential governing the collision of the reactants to depend upon the orientation of the HD molecule with respect to the approaching $\mathrm{Kr}^{+}$ion. At low collision energies, this angle-dependent term in the inter-

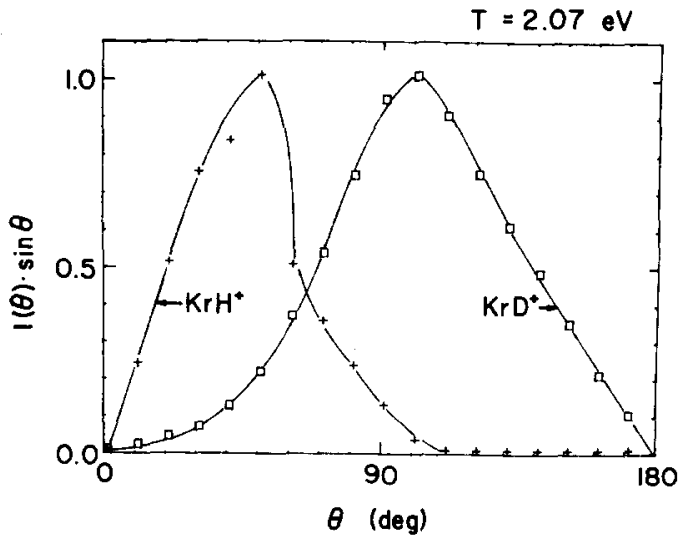

FIG. 14. Center-of-mass angular $(\theta)$ distributions for $\mathrm{KrH}^{+}$ (crosses) and $\mathrm{KrD}^{+}$(squares) produced in Reaction (2) at 2.07 $\mathrm{eV}$ initial relative energy. The two isotopic products show remarkably different angular distributions, with $\mathrm{KrH}^{+}$being confined to the forward hemisphere and $\mathrm{KrD}^{+}$being scattered predominantly through angles larger than $90^{\circ}$. 


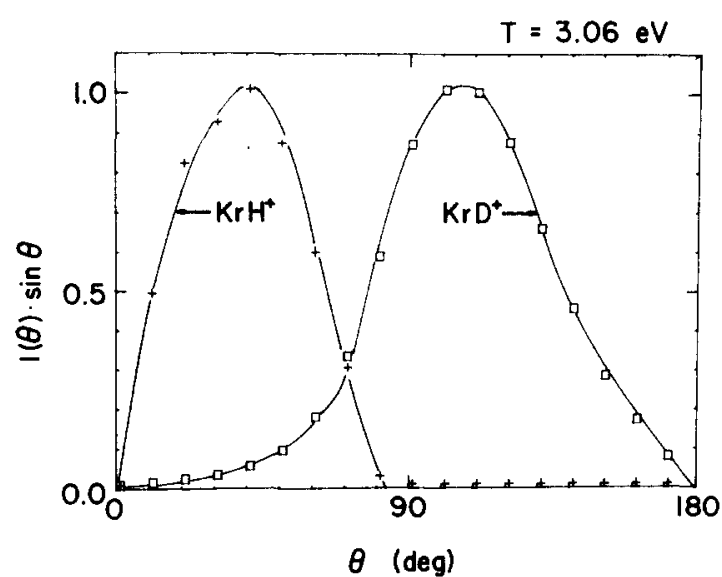

FIG. 15. Center-of-mass angular $(\theta)$ distributions for $\mathrm{KrH}^{+}$ (crosses) and $\mathrm{KrD}^{+}$(squares) produced in Reaction (2) at 3.06 $\mathrm{eV}$ initial relative energy. Note the extreme anisotropy of the two distributions.

molecular potential will enhance the probability that the $\mathrm{Kr}^{+}$ion collides with the hydrogen end of the HD molecule. At higher collision energies and concomitantly shorter interaction times, the inertia of the HD molecule prevents it from becoming oriented in the energetically most favorable position prior to collision; hence, the KIE would be expected to decrease to unity.

As mentioned above, the inverse isotope effect observed at collision energies greater than about $2 \mathrm{eV}$ (c. m. ) is very similar to the intramolecular isotope effect observed by Henchman and Johnson ${ }^{44}$ for the reaction of $\mathrm{O}^{+}$with $\mathrm{HD}$. Mahan and co-workers ${ }^{43}$ have studied the dynamics of the $\mathrm{O}^{+}+\mathrm{H}_{2}$ reaction and its isotopic variants. They interpreted their results at high energy ${ }^{43 b}$ in terms of a sequential impulse model in which product formation is regarded as resulting from two sequential two-body interactions. This model, while successful in explaining the dynamical features of the reaction, does not predict the observed inverse isotope effect at moderate collision energies $(T<6 \mathrm{eV})$ or the increase in the KIE at higher energies. However, their earlier discussion ${ }^{432}$ of general kinematic considerations in collinear collisions appears relevant to the discussion of this inverse isotope effect and is, therefore, summarized below.

It is generally known that the dynamics of a three atom collinear collision can be represented simply and correctly by the motion of a frictionless mass point sliding on a potential energy surface which has been plotted in a coordinate system which diagonalizes the kinetic energy. This diagonalization (carried out to avoid cross terms) causes the bond distance coordinates to be skewed through an angle $\beta$, given by

$$
\tan ^{2} \beta=m_{\mathrm{B}} m_{\mathrm{T}} / m_{\mathrm{A}} m_{\mathrm{C}},
$$

where $m_{\boldsymbol{i}}$ represents the mass of atom $i$ in the reaction $\mathrm{A}+\mathrm{BC} \rightarrow \mathrm{AB}+\mathrm{C}$, and where $m_{T}$ is the total mass. For Reaction (2), $\beta=55^{\circ}$ for formation of $\mathrm{KrD}^{+}$and $36^{\circ}$ for $\mathrm{KrH}^{+}$.

If the relative collision energy is sufficiently high, the trajectories are influenced only by the repulsive walls of the potential energy surface. The true surface may then be approximated by one corresponding to the interaction of two ideal hard spheres: two infinitely steep repulsive walls which intersect each other with an interior angle $\beta$. The available experimental evidence indicates that the actual $\mathrm{Kr}^{+} / \mathrm{HD}$ surface is quite flat: (1) the reaction is only slightly exothermic (by $0.3 \mathrm{eV}$ for ground-state reactants), with no measurable energy barrier in the entrance valley ${ }^{25}$ and no indication of a deep well corresponding to a $\mathrm{KrHD}^{+}$intermediate complex ${ }^{23}$; (2) the small reaction cross sections (relative to the $\mathrm{Ar}^{+} / \mathrm{H}_{2}$ system, for example) suggest that reactant attraction is rather weak and thus decreases the range of impact parameters leading to "close collisions"; (3) the product velocity vector distributions are nearly isotopic about the center of mass at low collision energies, suggesting that reaction occurs in rather centric collisions with hard sphere scattering of the product ion. ${ }^{27}$ Thus, it would appear that a discussion in terms of hard-sphere scattering may be appropriate in this system at collision energies as low as 2 or $3 \mathrm{eV}$.

Representing the collision dynamics by the motion of a frictionless mass point on the two skewed potential energy surfaces (and assuming specular reflection at the repulsive walls), one finds that $\mathrm{KrD}^{+}$is produced by a sequence of two hard-sphere collisions: $\mathrm{Kr}^{+}$strikes the $\mathrm{D}$ atom, D hits $\mathrm{H}, \mathrm{H}$ moves away from the newly formed $\mathrm{KrD}^{+}$(see Fig. 16). $\mathrm{KrH}^{+}$formation, on the other hand, requires a sequence of four hard-sphere collisions to reflect the particles out the product channel. Nearly collinear collisions are much more probable than exactly collinear ones, and a sequence of four hard-sphere colThis has two consequences: (1) the yield of backscattered $\mathrm{KrD}^{+}$is expected to be much greater than that of backscattered $\mathrm{KrH}^{+}$because of the simpler sequence of collisions required for $\mathrm{KrD}^{+}$production in collinear or nearly collinear encounters; (2) if, for whatever reasons, reaction is most efficient when the atoms are approximately collinear, there would be a significant inverse isotope effect favoring production of $\mathrm{KrD}^{+}$over $\mathrm{KrH}^{+}$at collision energies sufficiently high for the hardsphere approximation to be valid.

\section{B. Reaction dynamics}

Additional information on the nature of the reaction of $\mathrm{Kr}^{+}$with $\mathrm{HD}$ can be obtained by a comparison of the ve-

$$
\begin{array}{ll}
K r-D-H & K r-H-D \\
\beta=55^{\circ} & \beta=36^{\circ}
\end{array}
$$
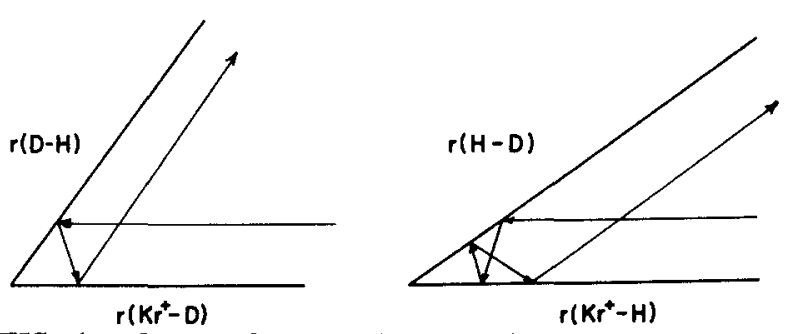

FIG. 16. Potential energy diagrams for the collinear interaction of ideal hard spheres. The angle $\beta$ at which the bond distance coordinates are skewed is given by Eq. (10). 
locity vector distributions for the two isotopic products. Figure 3 shows the distribution for $\mathrm{KrH}^{+}$ions formed in Reaction (2) at a collision energy of $0.678 \mathrm{eV}$ (c.m.), near which energy the KIE has its maximum value of about 2.5. The $\mathrm{KrH}^{+}$product ion is seen to be distributed in a nearly isotopic manner about the c.m. The other product of Reaction (2), $\mathrm{KrD}^{+}$, also displays a nearly isotropic distribution about the c.m. at this collision energy (Fig. 4). These two distributions are, within experimental uncertainty, identical to those previously reported ${ }^{27,28}$ for the reaction of $\mathrm{Kr}^{+}$with the homonuclear molecules $\mathrm{D}_{2}$ and $\mathrm{H}_{2}$ at approximately the same collision energy.

Product velocity vector distributions symmetric about the $\pm 90^{\circ}$ axis in the c.m. system would, of necessity, be produced if the reaction proceeded via the formation of a long-lived complex ${ }^{45}$; there is, however, no reason to suppose that the potential energy hypersurface for this reaction contains a basin of the depth necessary for the formation of such a complex. Moreover, if the putative $\mathrm{KrHD}^{+}$complex were indeed formed, it would preferentially decompose to $\mathrm{KrD}^{+}+\mathrm{H}$ because of the lower zeropoint energy of this ionic product. The observation that $\mathrm{KrH}^{+}$is the dominant product at this energy is taken as further evidence that Reaction (2) does not proceed via complex formation.

It is possible for certain types of direct interactions to yield product velocity vector distributions which peak at the c.m., and several such cases have already been described in the literature. ${ }^{23,27,46}$ In discussing the $\mathrm{Kr}^{+}\left(\mathrm{D}_{2}, \mathrm{D}\right) \mathrm{KrD}^{+}$reaction, for example, Henglein ${ }^{27}$ attributed the symmetry of the product distributions about the c.m. to hard-sphere scattering and strongly coupled interactions involving all three atoms. Such symmetric distributions have also been discussed in terms of the density of final translational states and the possibility that this density reaches a maximum at a velocity quite close to that of the c.m. ${ }^{46}$

We conclude, therefore, that Reaction (2) occurs at this energy by a direct mechanism in which all three atoms interact strongly enough to transfer appreciable momentum to the freed atom. There appears, however, to be little release to repulsive energy as the products separate, so that the product velocity vector distribution peaks very close to the $\mathrm{c} . \mathrm{m}$.

This conclusion is strengthened by the nature of the c. $m$. angular distributions for the two isotopic products at this energy (Fig. 12). These distributions show that, although appreciable back scattering of the ionic products occurs, complete symmetry about the $90^{\circ}$ axis is lacking. The angular distributions clearly peak in the forward direction, with the maximum in the polar differential cross section occurring at approximately $55^{\circ}$ for $\mathrm{KrD}^{+}$and $65^{\circ}$ for $\mathrm{KrH}^{+}$.

Velocity vector distributions for $\mathrm{KrH}^{+}$and $\mathrm{KrD}^{+}$produced at $1.41 \mathrm{eV}$ relative energy (where the $\mathrm{KIE} \cong 1$ ) are shown in Figs. 5 and 6, respectively. The two distributions resemble each other quite closely, as was the case at lower energies. The maximum product intensity is now clearly forward of the c.m. but there remains sig- nificant product intensity at large scattering angles. This is shown clearly in the c.m. angular distributions (Fig. 14), which maximize at about $40^{\circ}$ for $\mathrm{KrD}^{+}$and $50^{\circ}$ for $\mathrm{KrH}^{+}$, values somewhat smaller than those found at $0.678 \mathrm{eV}$ relative energy.

The asymmetry of the product distributions about the c. $m$. is clear proof that reaction is dominated by a direct mechanism at this energy; the occurrence of largeangle scattering and the observation that the most probable product velocity is considerably less than that expected on the basis of the spectator stripping model indicate that a moderately strong interaction still occurs between all three atoms, with some momentum being transferred to the freed atom.

The observation that the two isotopic products have quite similar velocity vector distributions contrasts sharply with the dynamic isotope effects reported for several other ion-molecule reactions which proceed by direct mechanism. In the reactions of $\mathrm{Ar}^{+}, \mathrm{N}_{2}^{+}$, and $\mathrm{O}^{+}$ with $\mathrm{HD}$, for example, the velocity vector distributions of the two isotopic products differ significantly. The reaction of $\mathrm{C}^{+}$with $\mathrm{HD}$, on the other hand, does give very similar isotopic product velocity vector distributions at collision energies less than $7 \mathrm{eV} .{ }^{46}$ The observation that the isotopic products of Reaction (2) have very similar distributions supports the idea that, at collision energies less than about $1.5 \mathrm{eV}$ (c.m.), this reaction is dominated by a mechanism which, while direct, nevertheless involves strong coupling between all three atoms.

The velocity vector distributions for the two isotopic products become significantly different, however, when the relative collision energy is increased to $2.07 \mathrm{eV}$ (at which energy the $\mathrm{KIE} \cong 0.5$ ). The $\mathrm{KrH}^{+}$distribution (Fig. 7) is confined almost entirely to the small-angle, glancing collision region, with the most probable product velocity being nearly equal to that predicted by the spectator stripping model. In contrast, the $\mathrm{KrD}^{+}$ions (Fig. 8) are found predominantly in the large-angle, rebound collision region, with the peak in the Cartesian probability distribution clearly located behind the c.m. The c.m. angular distributions (Fig. 14) dramatically demonstrate this anisotropy in product velocity vector distributions. The $\mathrm{KrH}^{+}$angular distribution is relatively narrow and peaks at about $50^{\circ}$. The $\mathrm{KrD}^{+}$distribution, however, is rather broad and is centered in the backward hemisphere; the most probable c.m. scattering angle is about $100^{\circ}$ at this energy, whereas it had been only $40^{\circ}$ at $1.41 \mathrm{eV}$ initial relative energy. It is thus this abrupt shift of the $\mathrm{KrD}^{+}$product from the forward to the backward hemisphere rather than any change in the $\mathrm{KrH}^{+}$scattering pattern which produces the extreme anisotropy in the velocity vector distributions of isotopic products above $2 \mathrm{eV}$ initial relative energy.

This unusual behavior becomes even more pronounced when the relative collision energy is increased to 3.06 $\mathrm{eV}$ (at which energy the $\mathrm{KIE} \cong 0.3$ ). The $\mathrm{KrH}^{+}$distribution (Fig. 9) is confined entirely to the forward hemisphere, with the peak occurring almost at the spectator stripping velocity. The $\mathrm{KrD}^{+}$distribution has continued its shift to the backward hemisphere, the position of maximum product probability now appearing well behind 
the c.m. The c.m. angular distributions (Fig. 15) shows the extreme anisotropy occurring at this energy: the rather narrow $\mathrm{KrH}^{+}$distributions peaks at $40^{\circ}$, while the slightly broader $\mathrm{KrD}^{+}$distribution peaks at about $115^{\circ}$.

The asymmetry of each product distribution about the $\pm 90^{\circ}$ axis indicates that the reaction proceeds by a direct mechanism at this energy. Such reactions, in general, exhibit a correlation between impact parameter and scattering angle, with glancing collisions causing little angular deflection and nearly head-on collisions producing large-angle scattering. ${ }^{47}$ The extreme anisotropy found at this energy, therefore, clearly implies that $\mathrm{KrH}^{+}$is produced primarily in large impact parameter, noncollinear collisions, whereas $\mathrm{KrD}^{+}$is produced preferentially in nearly collinear collisions. Because noncollinear collisions are generally more probable $[P(\theta) \propto \sin \theta$, where $\theta$ is the angle between the HD bond and the relative velocity vector], one might expect the increased anisotropy in the velocity vector distributions at higher collision energies to be accompanied by a steadily increasing isotope effect. Exactly the opposite is found experimentally, however, with the $\mathrm{KrD}^{+}$intensity being approximately 3 times the $\mathrm{KrH}^{+}$intensity at this energy. This remarkable result indicates that, for this particular system, nearly collinear collisions are much more likely to lead to atom transfer than are the generally more probable noncollinear collisions.

The tendency in reactions of this type for the hydrogen-containing product to be scattered through smaller angles than the deuterium-containing product (especially at the higher collision energies) has been observed to some extent in the reactions of $\mathrm{N}_{2}^{+}, \mathrm{O}_{2}^{+}$, and $\mathrm{O}^{+}$with $\mathrm{HD}^{13,43,48}$ and is presumably a characteristic of direct interaction processes. ${ }^{46}$ Extreme anisotropy of the kind reported here, although quite unusual, has been observed by Mahan and co-workers ${ }^{48-50}$ and subsequently confirmed by Henglein ${ }^{51}$ for the system $\mathrm{O}_{2}^{+}+\mathrm{HD}$ at relative collision energies above $6 \mathrm{eV}$. Mahan has discussed $^{52}$ three possible causes of such anisotropy in the product velocity vector distributions: (1) rotation of a short-lived collision complex, (2) stabilization of the nascent products by energy transfer to the freed atom, and (3) conservation of angular momentum and the difficulty of doing so when the reduced mass of the products is much smaller than that of the reactants.

Since the $\mathrm{Kr}^{+}+\mathrm{HD}$ system displays this extreme anisotropy at $2 \mathrm{eV}$, a collision energy well below the critical energy for product stability, this factor is not sufficient to explain the observed anisotropy. (Mahan reached essentially the same conclusion with regard to the $\mathrm{O}_{2}^{+}+\mathrm{HD}$ system. $)^{48}$ Because the products of Reaction (2a) have a larger reduced mass for relative translational motion than do the products of Reaction ( $2 b$ ), conservation of angular momentum would suggest that $\mathrm{KrH}^{+}$formation can occur at larger impact parameters than can $\mathrm{KrD}^{+}$ formation. As discussed above, however, the higher probability of large impact parameters should result in greater production of $\mathrm{KrH}^{+}$than $\mathrm{KrD}^{+}$, whereas the reverse is actually observed. Moreover, this proposed explanation would predict the same extreme anisotropy in the reaction of $\mathrm{Ar}^{+}$and $\mathrm{N}_{2}^{+}$with $\mathrm{HD}$. Since this effect is not observed in these reactions, we must conclude that conservation of angular momentum is not a determining factor in the dynamics of these hydrogen transfer reactions and is probably not the principal cause of the extreme anisotropy observed in the specific reaction considered here.

This extreme anisotropy is, however, exactly what one would expect from the general considerations govern ing the collinear collision of three atoms on a skewed potential energy surface appropriate to the interaction of ideal hard spheres (see preceding section). Thus, the inverse kinetic isotope effect and the anisotropy in the velocity vector distributions at collision energies above $2 \mathrm{eV}$ are both consistent with the conclusions that the $\mathrm{Kr}^{+} / \mathrm{HD}$ potential ene rgy surface is quite flat, and that nearly collinear collisions are much more likely to lead to reaction than are the generally more probable noncollinear collisions.

\section{SUMMARY}

The kinetic isotope effect for the exchange reaction of $\mathrm{Kr}^{+}$with $\mathrm{HD}$ exhibits a very unusual energy dependence. The ratio $\sigma\left(\mathrm{KrH}^{+}\right) / \sigma\left(\mathrm{KrD}^{+}\right)$inc reases rapidly with collision energy, passes through a maximum of about 2.5 at $0.7 \mathrm{eV}$ (c.m.), and then decreases to a value of about 0.3 at energies above $3 \mathrm{eV}$ (c.m.). This behavior is significantly different from that reported for the reactions of other simple ions (such as $\mathrm{Ar}^{+}, \mathrm{N}_{2}^{+}$, and $\mathrm{CO}^{+}$) with HD.

At low collision energies ( $<1 \mathrm{eV} \mathrm{c.m.)}$, the velocity vector distributions for $\mathrm{KrH}^{+}$and $\mathrm{KrD}^{+}$are very similar, being nearly isotropic about the center of mass. Reaction is thought to proceed by a direct mechanism in which all three atoms interact strongly enough to transfer appreciable momentum to the freed atom. At moderate collision energies ( $1-2 \mathrm{eV} \mathrm{c.m}$.), the distributions of the two isotopic products are still quite similar to each other but now peak somewhat forward of the center of mass, indicating that the reaction mechanism becomes increasingly impulsive as the collision energy is increased. At higher energies $(>2 \mathrm{eV})$, however, the velocity vector distributions of the isotopic products are dramatically different from each other: $\mathrm{KrH}^{+}$is strongly forward scattered (implying formation occurs predominantly in large impact parameter collisions), whereas $\mathrm{KrD}^{+}$is back scattered (small impact parameter collisions). This behavior, and the inverse isotope effect, appear to be a consequence of the relatively flat potential energy surface for this system and the requirement that collisions be collinear (or nearly so) for reaction to occur most efficiently.

\section{ACKNOWLEDGMENTS}

Acknowledgment is made to the donors of the Petrole um Research Fund, administered by the American Chemical Society, for partial support of this reasearch. Additional support was provided by the Research Corporation and by the University of Kansas General Research Fund. We would like to thank Professor Robert Sampson and the Kansas Geological Survey for providing the computer program used to construct the product velocity 
vector diagrams, and Professor Michael Henchman for his helpful suggestions.

\section{APPENDIX}

The distribution in relative collision energy caused by the thermal motion of the target gas molecules has been derived by Chantry ${ }^{37}$ for the case of a monoenergetic particle beam interacting with target molecules having an isotropic velocity distribution corresponding to temperature $T^{\circ} \mathrm{K}$. He showed that, provided the nominal c. m. energy $T_{0}$ exceeds a few $k T$, the distribution in relative collision energies has a full-width at half-maximum (FWHM) of

$$
\text { FWHM }=\left(11.1 \gamma k T T_{0}\right)^{1 / 2},
$$

where $\gamma=m_{\mathbf{A}} /\left(m_{\mathbf{A}}+m_{\mathbf{B C}}\right), m_{\mathbf{A}}$ and $m_{\mathrm{BC}}$ being the projectile and target masses, respecitively. A plot of Eq. (A1) appears as curve $a$ in Fig. 1.

The energy inhomogeneity of the primary ion bearn is known to increase with beam energy in a manner that can be approximated by the linear relation

$$
\Delta E=0.013 E+0.15 \mathrm{eV} \text {, }
$$

where $\Delta E$ is the energy spread (FWHM) and $E$ is the primary ion beam energy in the laboratory (LAB) system. ${ }^{29 a}$ The corresponding width of the c. $m$. energy distribution, ignoring target motion, is given by

$$
\begin{aligned}
\text { FWHM } & =(1-\gamma) \Delta E \\
& =0.013 T_{0}+0.15(1-\gamma) \mathrm{eV} .
\end{aligned}
$$

A plot of Eq. (A3) appears as curve $b$ in Fig. 1. Com parison of the two curves shows that thermal motion of the target causes a much greater spread in collision energies than does the energy inhomogeneity of the primary ion beam.

The velocity of the center of mass of the system, $\mathrm{v}_{\mathrm{c}_{\text {.m. }},}$, is given by

$$
\mathbf{v}_{c_{\cdot} m_{\bullet}}=\gamma \mathbf{v}_{A}+(1-\gamma) \mathbf{v}_{B C},
$$

where $v_{A}$ and $v_{B C}$ are the LAB velocities of the projectile and target, respectively. For a monoenergetic projectile beam interacting with a nonstationary target gas, the $x$ component of the velocity of the center of mass (i.e., the component parallel to $v_{A}$ ) will be distributed symmetrically about the nominal value $\gamma v_{\mathrm{A}}$, and with a full-width at half-maximum given by

$$
\text { FWHM }=2(1-\gamma) v_{\mathrm{BC}, 1 / 2},
$$

where $v_{\mathrm{BC}, 1 / 2}$ is the velocity of $\mathrm{BC}$ for which its one-dimensional distribution function has decreased to onehalf its maximum value. For a gas with a Maxwellian velocity distribution, it is readily shown that

$$
v_{\mathrm{BC}, 1 / 2}=0.824\left(2 k T / m_{\mathrm{BC}}\right)^{1 / 2} \text {. }
$$

Substitution of Eq. (A6) into (A5) gives the width in the distribution of c.m. velocities arising solely from the thermal motion of the target gas molecules. The result is presented as curve $a$ in Fig. 2 .

The width $\Delta v_{\mathrm{A}}$ of the projectile velocity distribution corresponding to a width $\Delta E$ in the LAB energy distribu- tion is given by

$$
\Delta v_{\mathrm{A}}=\left(2 / m_{\mathrm{A}} E\right)^{1 / 2} \Delta E
$$

Neglecting target motion, this inhomogeneity in projectile velocities would produce a distribution of c.m. velocities having a full-width at half-maximum given by

$$
\text { FWHM }=\gamma \Delta v_{A} \text {. }
$$

Substitution of Eqs. (A2) (after appropriate conversion from eV to ergs) and (A 7) into Eq. (A8) permits calculation of the width of the distribution of c.m. velocities produced solely by the spread in projectile velocities. The result, shown as curve $b$ in Fig. 2, indicates that this spread in projectile velocities produces a greater spread in c. $m$. velocities than does the thermal motion of the target molecules.

${ }^{1}$ F. S. Klein, Ann. Rev. Phys. Chem. 26, 191 (1975).

${ }^{2} J$. Dubrin, Ann. Rev. Phys. Chem, 24, 97 (1973).

${ }^{3}$ V. L. Talroze and G. V. Karachevtsev, Adv. Mass Spectrosc. 3, 211 (1966).

${ }^{4}$ E. W. McDaniel, V. Cermak, A. Dalgarno, E. E. Ferguson, and L. Friedman, Ion-Molecule Reactions (Wiley, New York, 1970), pp. 299-306.

${ }^{5}$ D. P. Stevenson and D. O. Schissler, J. Chem. Phys. 29, 282 (1958).

${ }^{6}$ F. S. Klein and L. Friedman, J. Chem. Phys. 41, 1789 (1964).

${ }^{7}$ R. W. Rozett and W. S. Koski, J. Chem. Phys. 49, 3737 (1968).

${ }^{8}$ M. T. Bowers and D. D. Elleman, J. Chem. Phys, 61, 4606 (1969).

${ }^{9}$ J. H. Futrell and F. P. Abramson, Adv. Chem. Ser. 58, 107 (1966).

${ }^{10}$ M. A. Berta, B. Y. Ellis, and W. S. Koski, J. Chem. Phys. 44, 4612 (1966); Adv. Chem. Ser. 68, 80 (1966).

${ }^{11}$ P. M. Hierl, Z. Herman, and R. Wolfgang, J. Chem. Phys. 53, $660(1970)$.

${ }^{12}$ (a) P. Fennelly, Ph. D. thesis, Brandeis University, 1972; (b) P. Fennelly, M. J. Henchman, and A. S. Werner (unpublished results).

${ }^{13}$ W. R. Gentry, E. A. Gislason, B. H. Mahau, and C. W. Tsao, J. Chem. Phys, 49, 3058 (1968).

${ }^{14} \mathrm{~A}$. Henglein, K. Lacmann, and B. Knoll, J. Chem. Phys. 48, 1048 (1965).

${ }^{15}$ R. J. Suplinskas, J. Chem. Phys, 49, 5046 (1968).

${ }^{16}$ J. C. Light and S. Chan, J. Chem. Phys. 51, 1008 (1969).

${ }^{17}$ T. F. George and R. J. Suplinskas, J. Chem. Phys. 51, 3666 (1969); ibid. 54, 1037, 1046 (1971).

${ }^{18}$ J. C. Light and J. Lin, J. Chem. Phys. 48, 3209 (1965).

${ }^{19}$ J. C. Tully, Ber. Bunsenges, Phys. Chem. 77, 557 (1973).

${ }^{20}$ P. J. Kuntz and A. C. Roach, J. Chem. Phys, 59, 6299 (1973).

${ }^{21}$ S. Chivalak and P. M. Hierl, Chem. Phys. Lett. 45, 99 (1977).

${ }^{22}$ P. J. Kuntz and A. C. Road, Trans. Faraday Soc. 11, 259 (1972).

${ }^{23}$ A. Henglein, J. Phys. Chem. 76, 3883 (1972).

${ }^{24}$ K. R. Ryan and I. G. Graham, J. Chem. Phys, 58, 4260 (1973).

${ }^{25}$ P. F. Fennelly, J. D. Payzant, R. S. Hemsworth, and D. K. Bohme, J. Chem. Phys. 60, 5115 (1974).

${ }^{26}$ R. D. Smith, D. L. Smith, and J. H. Futrell, Chem. Phys. Lett. 32, 513 (1975).

${ }^{27}$ G. Bosse, A. Ding, and A. Henglein, Z. Naturforsch. Teil A 26, 932 (1971).

${ }^{28}$ J. R. Wyatt, L. W. Strattan, and P. M. Hierl, J. Chem. Phys. 65, 1593 (1976).

${ }^{29}$ (a) P. M. Hierl, L. W. Strattan, and J. R. Wyatt, Int. J. Mass Spectrom. Ion Phys. 10, 385 (1973); (b) J. R. Wyatt, 
L. W. Strattan, S. Chivalak, and P. M. Hierl, J. Chem. Phys. 63, 4582 (1975).

${ }^{30}$ H. D. Hagstrum, Phys. Rev. 104, 309 (1956).

${ }^{31}$ For a more detailed discussion of this problem, see A. J. Masson, $\mathrm{Ph} . \mathrm{D}$. thesis, Brandeis University, 1970 .

${ }^{32}$ C. B. Moore, Natl. Bur. Stand. Circ. 467, Vol. II (1952).

${ }^{33}$ (a) H. P. Weise, H. U. Mittman, A. Ding, and A. Henglein, Z. Naturforsch. Teil A 26, 1122 (1971); (b) W. G. Rich, S. M. Bobbio, R. L. Champion, and L. D. Doverspike, Phys. Rev. A 4, 2253 (1971).

${ }^{34} \mathrm{G}$. Herzberg, Spectra of Diatomic Molecules (Van Nostrand, Princeton, NJ, 1950), p. 534.

${ }^{35}$ J. D. Payzant, H. I. Schiff, and D. K. Bohme, J. Chem. Phys. 63, 149 (1975).

${ }^{36}$ Reference 34, p. 532 .

${ }^{37}$ P. J. Chantry, J. Chem. Phys, 55, 2746 (1971).

${ }^{38} \mathrm{~J}$. R. Wyatt, L. W. Strattan, S. C. Snyder, and P. M. Hierl, J. Chem. Phys. 62, 2555 (1975).

${ }^{39} \mathrm{R}$. Wolfgang and R. J. Cross, J. Phys. Chem. 73, 743 (1969).

${ }^{40}$ A. Henglein, Adv. Chem. Ser. 58, 63 (1966), and referenced cited therein.

${ }^{41}$ H. M. Rosenstock, C. R. Mueller, M. B. Wallenstein, and
M. L. Vestal, U. S. Atomic Energy Commission Rep. No. J. L. L. $-650-3-7$, UC-23, Isotopes-Industrial Technology TW4500,15 th ed.

${ }^{42}$ J. C. Light, J. Chem. Phys. 40, 3221 (1964).

${ }^{43}$ (a) K. T. Gillen, B. H. Mahan, and J. S. Winn, J. Chem. Phys. 58, 5373 (1973); (b) ibid. 59, 6380 (1973).

${ }^{44} \mathrm{R}$. Johnson, Ph.D. thesis, Brandeis University, 1973.

${ }^{45}$ D. R. Herschbach, Adv. Chem. Phys. 10, 332 (1966).

${ }^{46}$ B. H. Mahan and T. M. Sloane, J. Chem. Phys. 59, 5661 (1973).

${ }^{47}$ See, for example, R. D. Levine and R. B. Bernstein, Molecular Research Dynamics (Clarendon, Oxford, 1974), pp. 7677.

${ }^{48}$ M. H. Chiang, B. H. Mahan, C. W. Tsao, and A. S. Werner, J. Chem. Phys. 53, 3752 (1970).

${ }^{49}$ M. H. Chiang, E. A. Gislason, B. H. Mahan, C. W. Tsao, and A. S. Werner, J. Phys. Chem. 75, 1426 (1971).

${ }^{50}$ E. A. Gislason, B. H. Mahan, C. W. Tsao, and A. S. Werner, J. Chem. Phys, 50, 5418 (1969).

${ }^{51} \mathrm{G}$. Bosse, A. Ding, and A. Henglein, Ber. Bunsenges. Phys. Chem. 75, 413 (1971).

${ }^{52}$ B. H. Mahan, Acc. Chem. Res. 3, 393 (1970). 